\title{
Fuzzy Evaluation of Agricultural Water Conservancy Facilities in Reuse
}

Shen Shan Han, School of Resource Environment and Historical Culture, Xianyang Normal University, Xianyang, China Amit Sharma, Department of Computer Science and Engineering, Chitkara University, Chandigarh, India

\begin{abstract}
It is increasingly important to reuse the wastewater conservancy facilities, which can further promote the development of agricultural economy. The basis of full investigation and research on the wastewater conservancy facilities in rural areas by using fuzzy mathematics method. This paper constructs a hierarchy model to evaluate the reuse value of wastewater conservancy facilities. The model conducts empirical research on the wastewater conservancy facilities in a certain farmland. The research results show that the transformation of abstract research into quantitative research, a scientific and reasonable practical research model, is established, which can evaluate the research object well. The comprehensive evaluation value of a certain farmland is 0.608 . The wastewater conservancy facilities in the farmland are in good condition and can be repaired by reconstruction; some economic benefits are obtained.
\end{abstract}

\section{KEYWORDS}

Abandoned Water Conservancy Facilities, Fuzzy Mathematics Theory, Reuse Evaluation

\section{INTRODUCTION}

With the development of modern agriculture, the state's investment in agriculture is increasing, and the most important goal of the Ministry of agriculture is to solve the "three rural" problems. Water sources are the primary factor in maintaining hygiene, enhancing industrial growth, increasing agricultural land and minimizing dry land areas. Thus, we believe that recycled waste water will be a short-term solution to the long term problem of water shortage that hinders the growth of all sectors of the country. Nowadays, there are various innovative treatments that have been used to restore wastewater. The effluents discharged from various factories, depending upon its chemical composition, have to be treated with appropriate treatment processes. The recovered water can either be potable or nonpotable (Zobel R. W. et al., 1999). For the purpose of biological safety the reclaimed water is reused as scenic water by use of purification processes (Zheng, X., et al., 2019) such as artificial wetland oxidation pond and similar advanced oxidation process (Zhang W., et al.,2004). In this case, some waste water therapy occurs (anaerobic digestion) when methane is exposed to energy and the rest of the remaining biodegradable materials (bio solids and nutrients) (Zhang, J., et al., 2018) available at the end of treatment are used as fertilizer in agricultural field. According to the relevant contents of the No.1 document of the Central Committee (Zamani, R., et al., 2020), China will complete

DOI: 10.4018/IJAEIS.2021070104

This article published as an Open Access article distributed under the terms of the Creative Commons Attribution License (http://creativecommons.org/licenses/by4.0/) which permits unrestricted use, distribution, and production in any medium, provided the author of the original work and original publication source are properly credited. 
the national key water conservancy facilities and water-saving transformation in the middle of this century. We should further make full use of the abandoned water conservancy facilities, improve the management and maintenance work, increase the investment in agricultural water conservancy, and build the modern agricultural irrigation project (Xue, L. I., et al., 2018). Due to the extensive development of agriculture in the initial stage, the original agricultural water conservancy measures cannot meet the current agricultural production needs to a certain extent. In addition, the old and scattered water conservancy facilities cannot give full play to their functions and economic benefits (Yadav J, et al., 2021). The reuse of agricultural water conservancy facilities is a very meaningful project, but it needs further analysis and evaluation to ensure that it can be fully utilized, and on the basis of maximum effective utilization, good economic benefits should be obtained (Wen-Bo F., et al., 2018).

\section{LITERATURE REVIEW}

Pescod investigated few case studies for wastewater and TWW reuse, recycle and their effects on soil, human health, and crop yield and production (Walaa A., et al.,. 2019). Al-Aama and Nakhla) investigated the cost of TWW reuse for landscape irrigation in Jubail, Saudi Arabia. Crook and Surampalli (Singh B., et al., 2008; Sharma A., et al., 2019) compared the criteria of TWW reuse in several States in the USA. Rathee G., et al., 2020) demonstrated that water conservation, reuse and recovery measures in the natural gas and crude oil sectors alone had the potential to conserve up to $222 \mathrm{MCM} /$ year of water in Saudi Arabia. The study showed that the increase in secondary wastewater treatment and reuse might result in substantial cost and energy savings for six inland cities, while an estimated $26 \%$ of urban water needs could be met by such TWW (Kajenthira et al. 2011). Qadir et al. (2010) summarized the production, treatment and irrigation by TWW in the Middle East and North Africa (MENA) region. The authors have shown that the MENA region has high potential of beneficial reuse of Treated waste water, while some of the major constraints for slow progress are as follows:

- Inadequate information on environmental and health impacts;

- Incomplete economic analysis;

- High costs and low returns of developing collection networks;

- Lack of wastewater treatment and reuse cost recovery mechanisms;

- Mismatch between water pricing and regional water scarcity (Wei-X. L., et al., 2008).

Wen Minghao, Huang Xiaomei and others first made a field survey on the current domestic agricultural abandoned water conservancy facilities. The collected relevant data, and then constructed a scientific evaluation model using fuzzy mathematical analysis principles to analyze the current situation of farmland water conservancy facilities reuse. In the research, in order to avoid the various influence factors of farmland water conservancy facilities reuse. The analytic hierarchy process (AHP) method is used to make fuzzy evaluation of various influence factors. To calculated and analyzed the weights of different factors, and applied them to the model (Liu Q. Y., 2020). Finally, the scientific research results are obtained by using the model and solving the parameters. It can be seen that the utilization of agricultural water conservancy facilities is the largest through the utilization of agricultural facilities (Jun, W., et al., 2018). Through the investigation and analysis of waste farmland facilities in China, combined with the characteristics of the utilization of rural waste water conservancy facilities in different areas. With the help of fuzzy mathematical analysis theory, established the evaluation model, and evaluated the farmland water conservancy facilities through the model. Through the weight calculation of various factors of water conservancy facilities utilization. The influencing factors of agricultural waste water conservancy facilities are studied in a quantitative way.The reasonable evaluation results are obtained based on the analytic hierarchy 
process (Eva M. et al., 2008). The research shows that the maximum subordinate value of the farmland water conservancy facilities is 0.607 . This value indicates that the reuse level of the farmland waste facilities is in a good state, and social benefits can be brought into play through transformation and restoration (Duque W. O., et al., 2007).

In this paper, through the investigation and analysis of farmland waste facilities, using the fuzzy mathematics evaluation method, the abstract research is quantitatively analyzed and evaluated. Through the evaluation of domestic farmland waste facilities reuse. In order to solve the problem of agricultural development, improve the quality of agricultural economy to provide scientific guidance, but also make full use of the original agricultural facilities, has important significance to protect the ecological environment. Figure 1 shows the various water resources.

\section{CONSTRUCTION OF HIERARCHICAL STRUCTURE MODEL}

However, there are many factors that affect the quality of agricultural facilities. On the other hand, it is necessary to further analyze the multiple factors that affect the quality of agricultural facilities. The method is the most simple and practical method to deal with such problems. In this paper, the fuzzy mathematics evaluation theory is applied in the study of waste farmland water conservancy facilities. The main research methods are analytic hierarchy process and evaluation set, that is, the objects need to be evaluated are treated hierarchically. Therefore, the highest goal is taken as the measurement index to study the reuse of waste farmland and water conservancy facilities. At the same time, the other indicators of each level are analyzed one by one, according to the relationship between each

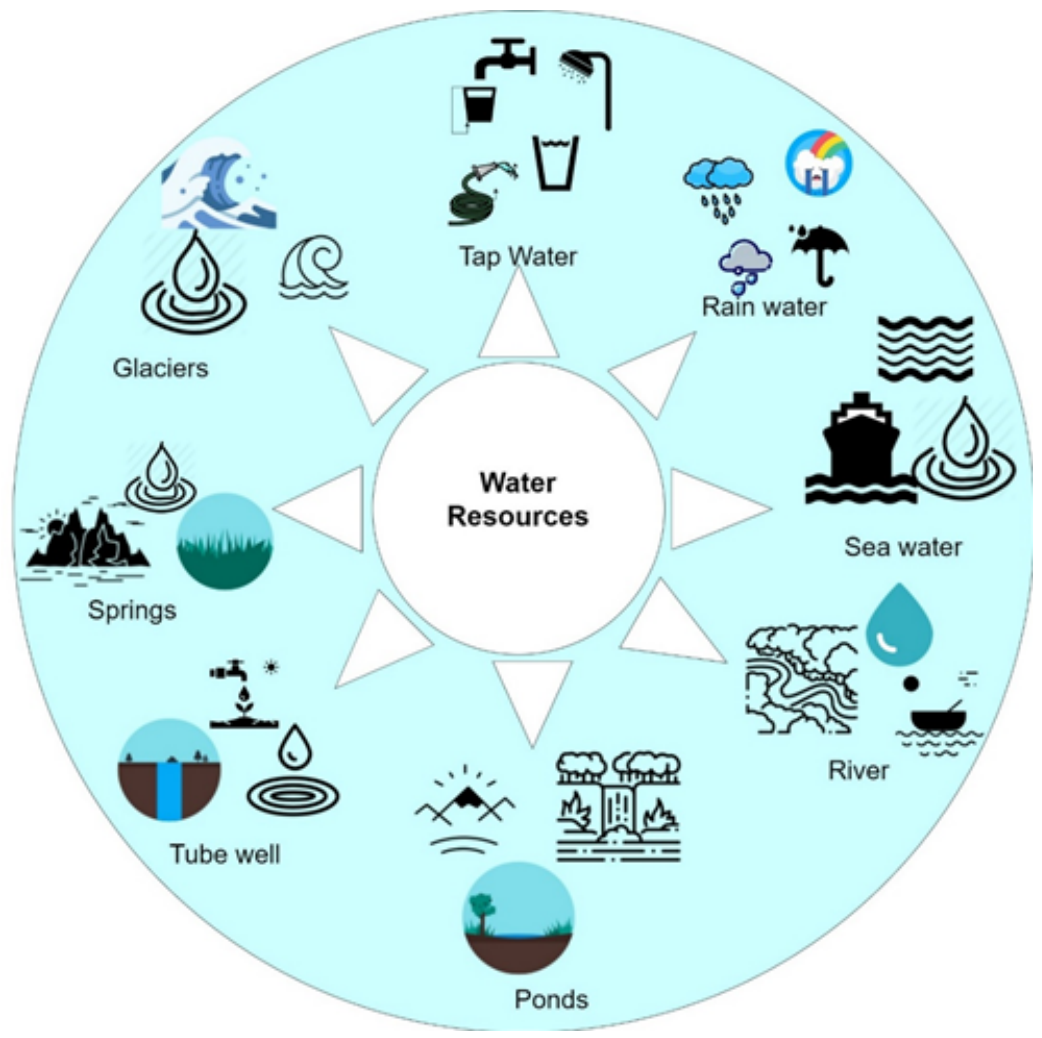


factor and membership, and then the factors affecting the reuse of facilities are combined to build a multi-level and objective research model (Deng-Feng, et al., 2019).

Through the above analysis, it is necessary to build multi-level, primary and secondary objectives for the reuse of farmland waste facilities, and establish a scientific and reasonable model to reflect the impact of each factor on the farmland waste facilities most truly and comprehensively. In the selection of influencing factors. We should try our best to consider the regional comprehensive development planning, project category, nature, current situation and benefit. Figure 2 shows the process for the treatment of waste water. Then according to the complexity and focus of the research object to construct the elements of each level. In the research, through field investigation and analysis and literature comparison, on the basis of full consideration of operability and authenticity, the following hierarchical model is constructed, as shown in Table 1.

According to the data in the table, it can be divided into three levels. The goal of the study is to reuse the abandoned farmland and water conservancy facilities. The first level index is the pumping station and reservoir, and the second level is the reuse benefit and maintenance degree. The three-level indicators in the model are evaluation indexes, among which u211-u224 are: water storage benefit, social investment, pump station reuse and repair investment, electrical equipment and auxiliary equipment, pump house and water pump; u111-u124 are main structure, auxiliary structure, operation and maintenance investment, storage pool repair investment, water storage efficiency and social investment.

In this paper, a water conservancy facility consists of four parts: pumping station, reservoir, inverted siphon and channel. Among them, the main body is brick structure, the reservoir is open reservoir, the pumps of pump station are all centrifugal pumps, the number is 1 . The channel is brick structure, inverted siphon is shaft type, and a 500mm deep grit chamber is set at the bottom. It is a square shaped shaft.

Figure 2. Process for the treatment of waste water

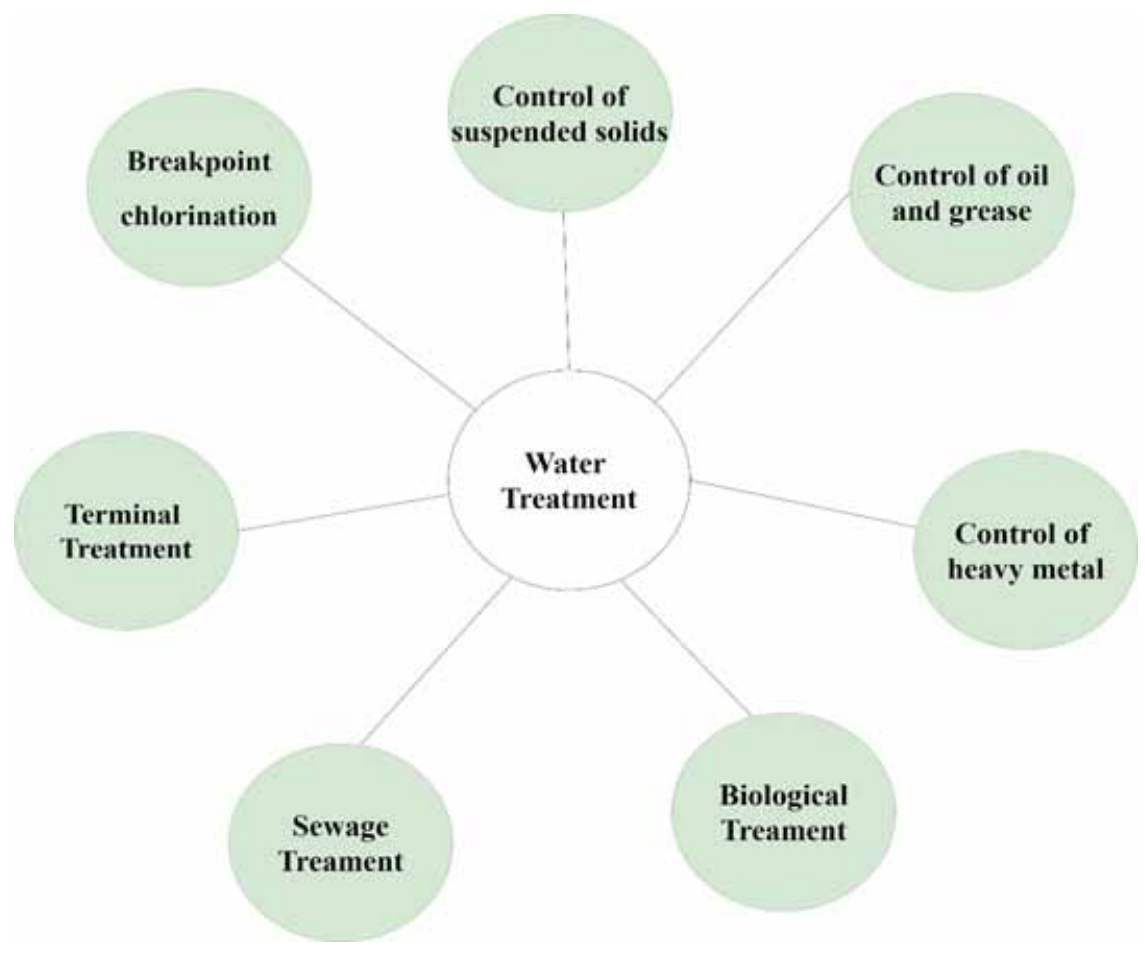


In order to facilitate the study, the set UI $=\{$ ui1, ui2... Is introduced It can be used as a fuzzy set for the evaluation of agricultural waste facilities, which satisfies $\cup \mathrm{Si}=1 \mathrm{ui}=\mathrm{u}$; UI $\cap \mathrm{UJ}=\Phi(\mathrm{I}$ $\neq \mathrm{J} ; \mathrm{I}, \mathrm{j}=1,2)$.

In the above set, UI refers to some engineering structures such as water storage project and water transmission and distribution project, and UIM is the main factor set affecting the indexes. Then, according to the relevant literature and reference evaluation, the evaluation fuzzy set $V=\{\mathrm{V} 1$, V2... Is established in order to truly reflect the utilization efficiency, combined with the regional characteristics, further establish the evaluation set $\mathrm{V}=\{\mathrm{V} 1, \mathrm{~V} 2, \mathrm{~V} 3, \mathrm{~V} 4, \mathrm{~V} 5\}$, that is, $\mathrm{v}=\{$ very poor, poor, medium, good, excellent $\}$ \}.

Determine the weight of the index. To evaluate each index reasonably, it is necessary to assign a value to each index and calculate its weight. As an important process in AHP, weight assignment plays an important role in the process of fuzzy comprehensive evaluation. However, in order to ensure the objectivity and effectiveness of the evaluation structure, it is necessary to reasonably consider the evaluation index. Based on the expert meeting method, empirical method, allicin average method, Delphi method and the combination of quantitative and qualitative methods, the final weight value is given.

This paper has selected the waste water conservancy facilities in a certain area as the research object, and the COD (chemical oxygen demand) content in the waste water conservancy facilities. Figure 3 shows the analysis model of reuse of abandoned farmland and water conservancy facilities. This paper will establish the actual fuzzy evaluation model from the following three aspects, and get the specific quantitative value. First, according to the actual situation of the farmland waste facilities to build a model, and discuss the importance of the influencing factors of each level index, and through pairwise comparison to determine its importance, the judgment matrix between the first level index and the second level index is obtained. Then, according to the eigenvector and the corresponding eigenvalue of each judgment matrix. In order to avoid the reliability of assigned weight data, consistency test should be carried out. In order to make the research scientific and reliable, we should fully consider the importance of objective, comprehensive and systematic factors affecting

Table 1. Analysis model of reuse of abandoned farmland and water conservancy facilities

\begin{tabular}{|c|c|c|c|}
\hline Evaluation objectives & Local works & Features of elements & $\begin{array}{l}\text { Evaluating } \\
\text { indicator }\end{array}$ \\
\hline \multirow{14}{*}{$\begin{array}{l}\text { Analysis on reuse of } \\
\text { abandoned farmland } \\
\text { and water conservancy } \\
\text { facilities }\end{array}$} & \multirow{8}{*}{ Pump station U2 } & \multirow{4}{*}{ Reuse benefits U 21} & U224 \\
\hline & & & $\mathrm{U} 223$ \\
\hline & & & U222 \\
\hline & & & $\mathrm{U} 221$ \\
\hline & & \multirow{4}{*}{ Maintenance level U22 } & U214 \\
\hline & & & U213 \\
\hline & & & $\mathrm{U} 212$ \\
\hline & & & $\mathrm{U} 211$ \\
\hline & \multirow{6}{*}{ Reservoir U1 } & \multirow{4}{*}{ Reuse benefits U12 } & U124 \\
\hline & & & U123 \\
\hline & & & U122 \\
\hline & & & U121 \\
\hline & & \multirow{2}{*}{ Maintenance level U11 } & U112 \\
\hline & & & U111 \\
\hline
\end{tabular}


Figure 3. Analysis model of reuse of abandoned farmland and water conservancy facilities

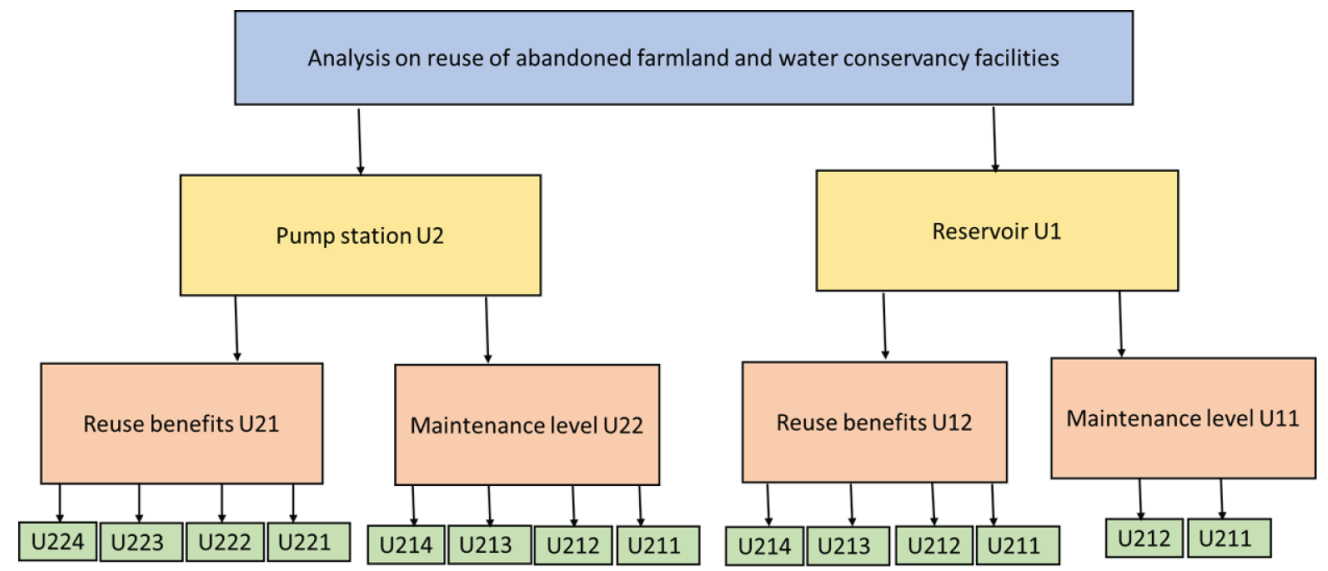

the reuse value of farmland waste facilities in the actual application process. Figures 4, 5 and 6 show the COD variation with year for entrance, center and exit of the waste water conservancy facility.

Through consulting the experienced experts, the decision-making problem of complex factors is preliminarily made, and the differences between the same indexes of different projects or different levels are discussed, and the determination is made according to the actual situation. In practical application, if the weak existence data does not meet the consistency test, it needs to be further tested by the following conditions. By integrating the influencing factors into $\mathrm{U}_{\mathrm{ij}}(\mathrm{J}=1,2 \mathrm{Further}$ analysis and evaluation were carried out with single factor evaluation, and the rank VK $(\mathrm{k}=1,2, \ldots)$ was obtained at the same time, the membership degree $\mathrm{R}_{\mathrm{ijk}}$ was determined. $\mathrm{r}_{\mathrm{ij}}=\left(\mathrm{r}_{\mathrm{ij} 1}, \mathrm{r}_{\mathrm{ij} 2}, \ldots\left(\mathrm{R}_{\mathrm{ijn}}\right)\right.$ denotes the single factor evaluation set of the $\mathrm{j}^{\text {th }}$ factor in the set UI.Therefore, the following fuzzy evaluation matrix can be constructed:

Figure 4. COD variation with year for entrance of the waste water conservancy facility

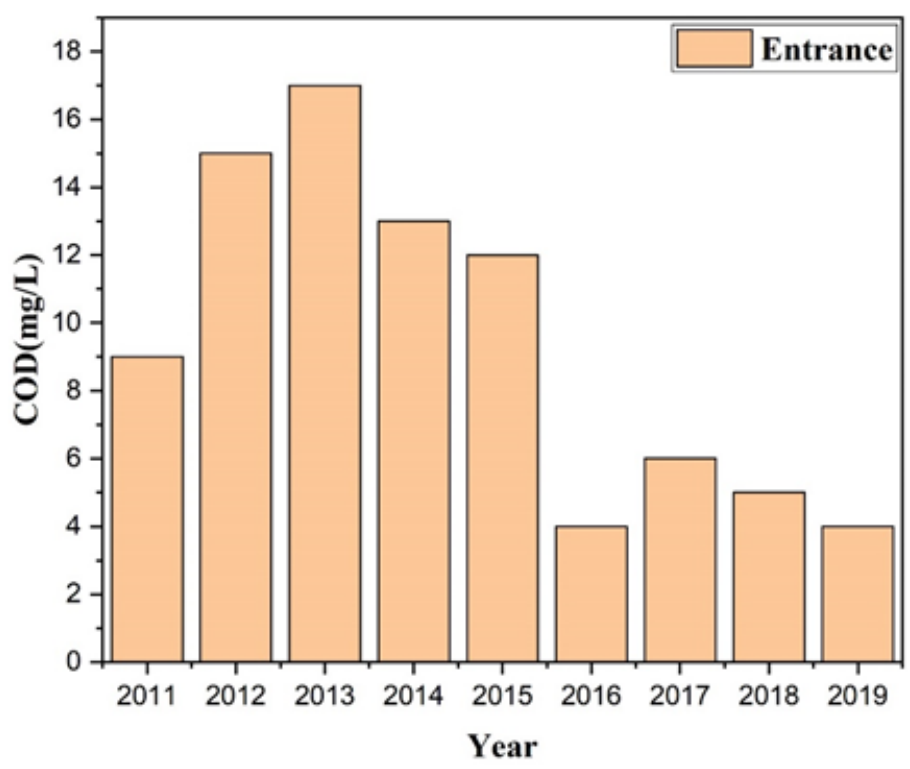


Figure 5. COD variation with year for center of the waste water conservancy facility

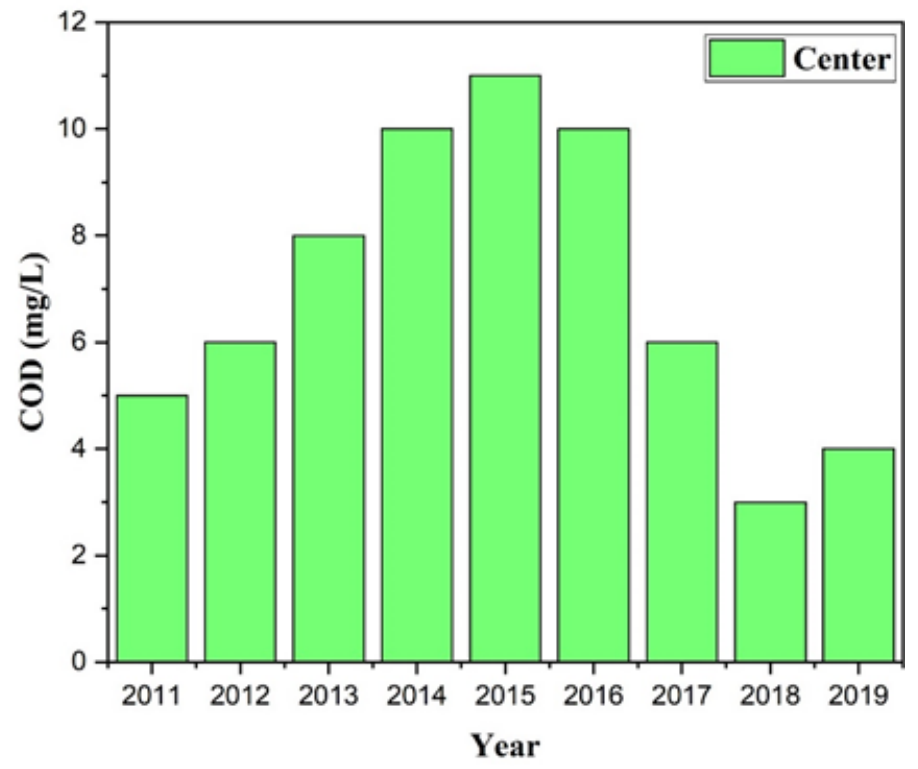

Figure 6. COD variation with year for center of the waste water conservancy facility

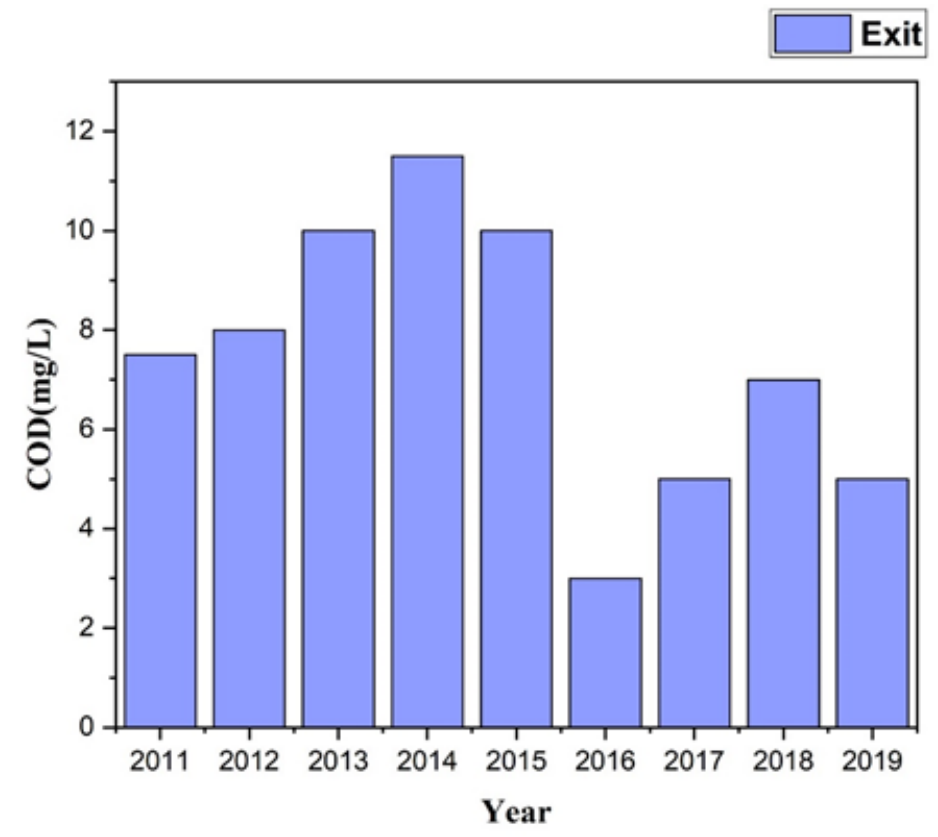




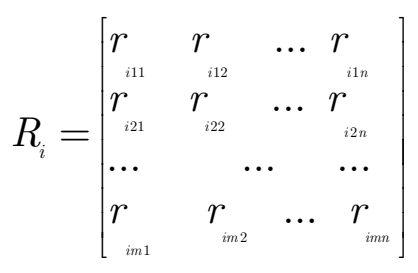

For membership degree, fuzzy statistical method can be used to determine the specific value, set $\mathrm{Rij}=\operatorname{mijjn}(\mathrm{J}=1,2,5), \mathrm{N}$ and $\mathrm{mij}$ in represent the number of experts participating in this session and the number of experts who think UI belongs to VJ.In order to ensure the reliability and stability of the evaluation results, the following aspects need to be considered.Firstly, the nature of the problem and the comprehensive analysis of related work as well as each factor and its internal relationship should be investigated; secondly, the number of experts should be appropriate; thirdly, the research materials and professional knowledge in this field need to be fully understood and mastered.Then use the method of fuzzy evaluation of each factor.Membership matrix and comment set should be considered in the operation:

$$
\underline{B_{i}}=W_{i} \times r_{i}=\left(b_{i 1}, b_{i 2} \ldots, b_{i n}\right) ;(i=1,2, \ldots, s)
$$

Then, combined with the above fuzzy evaluation results, a two-level fuzzy evaluation is carried out, and the evaluation matrix of single factor evaluation index is constructed:

$$
R=\left[\begin{array}{cccc}
b_{11} & b_{12} & \ldots & b_{1 n} \\
b_{21} & b_{22} & \ldots & b_{2 n} \\
\ldots & \ldots & \ldots & \ldots \\
b_{s 1} & r_{s 2} & \ldots & r_{s n}
\end{array}\right]
$$

The total evaluation matrix of the evaluation object in this study can determine the membership degree of the first level factors through the evaluation set, and the calculation method is as follows (4): the evaluation matrix of the evaluation object in this study can be determined by the membership degree of the first level factors:

$$
\underline{B}=W \times R=\left(b_{1}, b_{2}, \ldots, b_{n}\right)
$$

In the above formula (4), the final fuzzy evaluation result is $\underline{B}$. The value can be determined by the maximum membership degree problem in fuzzy mathematics theory, that is, the evaluation value of farmland waste facilities reuse can be obtained. When it is necessary to convert the calculated scalar value, the following formula (5) can be used:

$$
E=\bar{V} \times B^{T}
$$

In formula (5), the result set after quantifying the factors of comment set $\mathrm{V}$ is $\bar{V}$, this method, the fuzzy evaluation results of the highest level can be obtained step by step. In order to clearly and intuitively reflect the results of this study, this paper uses 5 scales to illustrate 
the problem, that is, the rating language set mentioned above, that is, the research results are divided into five grades, and the corresponding relationships are very poor (V5), poor (V4), medium (V3), good (V2), excellent (V1).

\section{EMPIRICAL ANALYSIS}

\subsection{Empirical Analysis}

Through the above analysis and calculation, the article calculates the weight value of each index of the research object, as shown in Table 2. Then, the membership degree of each index is further calculated.

Table 2. Weight results of abandoned farmland and water conservancy facilities

\begin{tabular}{|c|c|c|c|c|c|}
\hline $\begin{array}{l}\text { Main evaluation } \\
\text { factors }\end{array}$ & weight & $\begin{array}{c}\text { Secondary } \\
\text { evaluation } \\
\text { factors }\end{array}$ & weight & $\begin{array}{c}\text { Three level } \\
\text { evaluation factors }\end{array}$ & weight \\
\hline \multirow{8}{*}{ U1 } & \multirow{8}{*}{0.201} & \multirow{4}{*}{ U21 } & \multirow{4}{*}{0.375} & U214 & 0.14 \\
\hline & & & & U213 & 0.11 \\
\hline & & & & U212 & 0.43 \\
\hline & & & & U211 & 0.32 \\
\hline & & \multirow{4}{*}{$\mathrm{U} 22$} & \multirow{4}{*}{0.625} & U224 & 0.22 \\
\hline & & & & U123 & 0.28 \\
\hline & & & & U122 & 0.25 \\
\hline & & & & U121 & 0.25 \\
\hline \multirow{6}{*}{ U2 } & \multirow{6}{*}{0.285} & \multirow{4}{*}{ U12 } & \multirow{4}{*}{0.400} & U124 & 0.24 \\
\hline & & & & U123 & 0.27 \\
\hline & & & & U122 & 0.23 \\
\hline & & & & U121 & 0.26 \\
\hline & & \multirow{2}{*}{ U11 } & \multirow{2}{*}{0.600} & U112 & 0.24 \\
\hline & & & & U111 & 0.76 \\
\hline \multirow{8}{*}{ U3 } & \multirow{8}{*}{0.125} & \multirow{4}{*}{ U42 } & \multirow{4}{*}{0.400} & U421 & 0.25 \\
\hline & & & & U422 & 0.23 \\
\hline & & & & U423 & 0.28 \\
\hline & & & & U424 & 0.24 \\
\hline & & \multirow{4}{*}{ U41 } & \multirow{4}{*}{0.600} & U224 & 0.22 \\
\hline & & & & U123 & 0.28 \\
\hline & & & & U122 & 0.25 \\
\hline & & & & U121 & 0.25 \\
\hline \multirow{6}{*}{$\mathrm{U} 4$} & \multirow{6}{*}{0.416} & \multirow{4}{*}{ U32 } & \multirow{4}{*}{0.250} & U324 & 0.27 \\
\hline & & & & U323 & 0.31 \\
\hline & & & & U322 & 0.17 \\
\hline & & & & U321 & 0.23 \\
\hline & & \multirow{2}{*}{ U31 } & \multirow{2}{*}{0.750} & U312 & 0.29 \\
\hline & & & & U311 & 0.71 \\
\hline
\end{tabular}


Combining with the above formula (1) and formula (2), the result in Table 3 can be calculated, that is, the final rating result.

\subsection{Fuzzy Evaluation}

Through the above calculation results, we can get the first level fuzzy evaluation of engineering quality, the index weight and its relation matrix, and then calculate the comprehensive evaluation results of the project quality and indicators:

Table 3. Evaluation results of some abandoned farmland and water conservancy facilities

\begin{tabular}{|c|c|c|c|c|c|c|c|}
\hline \multirow[b]{2}{*}{ Main evaluation factors } & \multirow{2}{*}{$\begin{array}{c}\text { Secondary } \\
\text { evaluation factors }\end{array}$} & \multirow{2}{*}{$\begin{array}{c}\text { Three level } \\
\text { evaluation factors }\end{array}$} & \multicolumn{5}{|c|}{ Evaluation results } \\
\hline & & & $\begin{array}{l}\text { Very } \\
\text { bad }\end{array}$ & Poor & in & good & excellent \\
\hline \multirow{8}{*}{ U2 } & \multirow{4}{*}{$\mathrm{U} 21$} & U214 & 0.1 & 0.3 & 0.5 & 0.1 & 0 \\
\hline & & $\mathrm{U} 213$ & 0.1 & 0.1 & 0.2 & 0.6 & 0 \\
\hline & & $\mathrm{U} 212$ & 0 & 0.3 & 0.6 & 0.1 & 0 \\
\hline & & $\mathrm{U} 211$ & 0 & 0.2 & 0.7 & 0.1 & 0 \\
\hline & \multirow{4}{*}{$\mathrm{U} 22$} & U224 & 0 & 0.1 & 0.2 & 0.7 & 0 \\
\hline & & U223 & 0 & 0.1 & 0.3 & 0.6 & 0 \\
\hline & & U222 & 0 & 0.2 & 0.2 & 0.6 & 0 \\
\hline & & $\mathrm{U} 221$ & 0.1 & 0.2 & 0.5 & 0.2 & 0 \\
\hline \multirow{6}{*}{$\mathbf{U} 1$} & \multirow{4}{*}{ U12 } & U124 & 0 & 0 & 0.2 & 0.7 & 0.1 \\
\hline & & U123 & 0 & 0 & 0.1 & 0.8 & 0.1 \\
\hline & & U122 & 0 & 0.1 & 0.1 & 0.8 & 0 \\
\hline & & U121 & 0 & 0.1 & 0.1 & 0.8 & 0 \\
\hline & \multirow{2}{*}{ U11 } & U112 & 0.1 & 0.1 & 0.1 & 0.7 & 0 \\
\hline & & U111 & 0 & 0.1 & 0.2 & 0.7 & 0 \\
\hline \multirow{8}{*}{ U4 } & \multirow{4}{*}{$\mathrm{U} 42$} & $\mathrm{U} 421$ & 0 & 0 & 0.4 & 0.6 & 0 \\
\hline & & U422 & 0 & 0 & 0.3 & 0.6 & 0.1 \\
\hline & & U423 & 0 & 0 & 0.4 & 0.6 & 0 \\
\hline & & U424 & 0 & 0.2 & 0.2 & 0.6 & 0 \\
\hline & \multirow{4}{*}{ U41 } & U414 & 0 & 0 & 0 & 0.6 & 0.4 \\
\hline & & U413 & 0 & 0 & 0.3 & 0.6 & 0.1 \\
\hline & & U412 & 0 & 0 & 0.2 & 0.6 & 0.2 \\
\hline & & U411 & 0 & 0 & 0 & 0.6 & 0.4 \\
\hline \multirow{6}{*}{ U3 } & \multirow{4}{*}{ U32 } & U324 & 0 & 0 & 0.2 & 0.7 & 0.1 \\
\hline & & U323 & 0 & 0 & 0.2 & 0.7 & 0.1 \\
\hline & & U322 & 0 & 0 & 0.2 & 0.6 & 0.2 \\
\hline & & U321 & 0 & 0 & 0.3 & 0.7 & 0 \\
\hline & \multirow{2}{*}{ U31 } & U312 & 0 & 0.1 & 0.3 & 0.6 & 0 \\
\hline & & U311 & 0 & 0.1 & 0.1 & 0.7 & 0 \\
\hline
\end{tabular}


$\underline{B_{11}}=W_{11} * \underline{R_{11}}=(0,0.7,0.176,0.1,0.024)$

In the same way, the following results are obtained:

$\underline{B_{12}}=(0.0517,0.944,0.124,0.049)$

Then, the comprehensive evaluation of secondary indicators is calculated as follows:

$$
\begin{aligned}
& \underline{B_{1}}=W_{1} * \underline{R_{1}}=W_{1} *\left[\frac{B_{11}}{B_{12}}\right] \\
& =(0.021,0.798,0.110,0.028,0.042)
\end{aligned}
$$

The results are as follows:

$$
\begin{aligned}
& \frac{B_{2}}{B_{3}}=(0,0.292,0.472,0.221,0.025) \\
& \underline{B_{4}}=(0.023,0.671,0.173,0.075,0.053)
\end{aligned}
$$

On the basis of the comprehensive evaluation of the secondary index, the comprehensive evaluation result of the third level index is calculated by the following formula. The formula is as follows:

$$
\underline{B}=W_{1} * \underline{R}=W *\left[\begin{array}{l}
\frac{B_{1}}{B_{2}} \\
\frac{B_{3}}{B_{4}}
\end{array}\right]=(0.023,0.608,0.237,0.091,0.038)
$$

According to the calculation results, combined with the fuzzy identification method, according to its principle, we can know that the maximum membership value of the reuse value of the farmland waste water conservancy facilities is the maximum value in the matrix $\mathrm{B}$, that is, 0.608. Combined with the evaluation level established above, we can know that the farmland waste water conservancy facilities are in a good state, that is to say, they can play their functions through repair, It will produce certain economic benefits.

\subsection{Result Analysis and Discussion}

Through the study, we can see that the current farmland waste water conservancy facilities are better in regional selection and engineering quality, especially in the infrastructure such as channels and reservoirs, whose construction project quality is good, and can still be used normally after simple maintenance after years of abandonment. Combined with the results of the previous study, the weight of each parameter index in the engineering aspect is relatively large,For example, the damage degree of the main structure of the reservoir and the damage degree of the pump station are 0.600 and 0.625 respectively, which reflects that the construction quality of water conservancy facilities is not serious, 
and it will obviously reduce the investment in the later stage of repair. In addition, combined with the actual situation, the construction site of these existing waste water conservancy facilities is demonstrated by the early designers through a large amount of data, and there is no need to re select the site.

Then, considering the possibility of repair and the problems faced by the repair, the weight values of the recovery and utilization benefits of the pump station and the reservoir are 0.375 and 0.400 . According to the comments set above, the benefit of reuse is in the medium level. Because of the long-term idle, some electrical equipment such as the equipment in the pump room cannot be reused or repaired. In most cases, they are all replaced, the initial maintenance and treatment need to invest a certain amount of money, so the benefit will be reduced. In particular, with the change of agricultural demand, some places need to be reinforced, and a large number of repair materials need to be purchased again. The use of advanced repair methods requires a certain amount of manpower and material cost. However, considering the social and economic benefits in the later stage, the investment in the early stage is reasonable.

Finally, according to the results of various indicators and comprehensive evaluation, it can be seen that the reason for the low benefit of rehabilitation is the reconstruction of existing water conservancy facilities, and the simple repair investment is less.However, for water conservancy facilities in different regions, due to different demand, some water conservancy facilities need to be expanded and reconstructed to meet various national standards, which were not considered in the previous design, especially the implementation of the new standards.In addition, due to the change of water resources in agricultural irrigation areas, the existing facilities need to be reasonably transformed, and the traditional channels should be designed for dredging, anti-corrosion and anti-seepage, so as to reduce the loss of water resources in circulation. Especially for long-distance transportation, great repair work needs to be done, which leads to the increase of human costs. All these eventually lead to the investment in the whole restoration project. The overall reuse efficiency is low.

\section{CONCLUSION}

Based on the investigation of the waste water conservancy facilities in farmland, this paper makes quantitative analysis and calculation on the reuse of the waste water conservancy facilities. By combining the fuzzy evaluation method and the analytic hierarchy process, the paper makes a real, objective, scientific and reasonable comparison of the various influencing factors of the reuse of the waste water conservancy facilities. But the final results can truly reflect the impact of each index. The analysis results show that:

- Through the comprehensive analysis of water conservancy facilities, we can use the method of fuzzy structure analysis and comprehensive utilization;

- The research results show that the reuse value of some farmland waste water conservancy facilities is in a good state, the value is 0.608 , and certain economic benefits can be obtained by repairing the water conservancy facilities;

- The detailed results show that the abandoned irrigation and water conservancy facilities have certain advantages in engineering quality and region, but they are insufficient in management quality and maintenance. Most of the farmland water conservancy facilities can play their functions and functions after simple maintenance.

\section{ACKNOWLEDGMENT}

This research has been financed by the Research Base of philosophy and Social Sciences in Shaanxi Provincial Department of Education "Study on land use and landscape pattern change in Guanzhong Emperor Mausoleum of Han and Tang dynasties"(17JZ079). 


\section{REFERENCES}

Adhikari, A., Chhetri, V. S., \& Camas, A. (2020). Evaluation of microbiological quality of agricultural water and effect of water source and holding temperature on the stability of indicator organisms' levels by seven U.S. environmental protection agency-approved methods. Journal of Food Protection, 83(2), 249-255. doi:10.4315/0362-028X.JFP-19-381 PMID:31944139

Bai, Bouwmeester, \& Mohan. (2009). Fuzzy Logic Water Quality Index and Importance of Water Quality Parameters. Air, Soil and Water Research, 2. 10.4137/ASWR.S2156

Bo, W., Han, W. U., Tianyu, F., Yitong, D., \& Xiangtian, N. (2019). Evaluation of management level of water conservancy project supervision unit based on combination weight and fuzzy theory. Paper Asia, 35(1), 50-52.

Dahiya, S., Singh, B., Gaur, S., Garg, V. K., \& Kushwaha, H. S. (2007). Analysis of groundwater quality using fuzzy synthetic evaluation. Journal of Hazardous Materials, 147(3), 938-946. doi:10.1016/j.jhazmat.2007.01.119 PMID:17337324

Darbra, R. M., Eljarrat, E., \& Barceló, D. (2008). How to measure uncertainties in environmental risk assessment. Trends in Analytical Chemistry, 27(4), 377-385. doi:10.1016/j.trac.2008.02.005

Deng-Feng, W. U., Zhen-Zhen, R., \& Chun-Hua, G. E. (2019). Evaluation of soil fertility quality based on fuzzy mathematical model. Water Conservancy and Technology and Economy.

Duque, W. O. (2006). Assessing water quality in rivers with fuzzy inference systems: A case study. Environment International, 32(6), 733-742. doi:10.1016/j.envint.2006.03.009 PMID:16678900

Duque, W. O. (2007). Domingo. A neural-fuzzy approach to classify the ecological status in surface waters. Environmental Pollution, 148(2), 634-641. doi:10.1016/j.envpol.2006.11.027 PMID:17254680

Jun, W., Sen, Z., Shi-Ming, G., Li, Z., Business, S. O., \& University, H. A. (2018). The investigation of the agricultural water-saving willingness in the upper reaches of baiyang lake under the background of xiong'an new area. Management \& Technology of SME.

Kamal, J. (2019). Principal Component Analysis for Water Quality Assessment of the Ganga River in Uttar Pradesh, India. Water Resources, 46(5), 789-806. doi:10.1134/S0097807819050129

Kumar, D., Sharma, A., Kumar, R., \& Sharma, N. (2019). Restoration of the Network for Next Generation (5G) Optical Communication Network. 2019 International Conference on Signal Processing and Communication (ICSC), 64-68. doi:10.1109/ICSC45622.2019.8938337

Kumar, J. M., \& Ankit Shekhar, M. (2020). Assessing groundwater quality for drinking water supply using hybrid fuzzy-GIS-based water quality index. Water Research, 179, 115867. doi:10.1016/j.watres.2020.115867 PMID:32408184

Lifeng, Z. (2019). Study on the construction and management and protection of agricultural water conservancy project. Jiangxi Building Materials.

Liu, Q. Y., Wang, M. W., Dong, H., Shen, F. Q., \& Jin, J. L. (2020). A novel evaluation model for heavymetals pollution in soil based on connection numbers and Dempster-Shafer theory. International Journal of Environmental Science and Technology, 17(1), 541-552. doi:10.1007/s13762-019-02453-w

López, E. M., García, M., Schuhmacher, M., \& Domingo, J. L. (2008). A fuzzy expert system for soil characterization. Environment International, 34(7), 950-958. doi:10.1016/j.envint.2008.02.005 PMID:18378310

Rathee, G., Sharma, A., Kumar, R., Ahmad, F., \& Iqbal, R. (2020). A trust management scheme to secure mobile information centric networks. Computer Communications, 151, 66-75. doi:10.1016/j.comcom.2019.12.024

Recai Oktaş, C. (2020). Evaluation of quality of groundwater in irrigation using fuzzy logic in the Bafra Plain, Northern Turkey. Eurasian Journal of Soil Science, 9(4), 339-348. doi:10.18393/ejss.783470

Sharma, A., Kumar, R., \& Kaur, P. (2019). Study of Issues and Challenges of Different Routing Protocols in Wireless Sensor Network. Fifth International Conference on Image Information Processing (ICIIP), 85-590, doi:10.1109/ICIIP47207.2019.8985915 
Singh, B., Dahiya, S., Jain, S., Garg, V. K., \& Kushwaha, H. S. (2008). Use of fuzzy synthetic evaluation for assessment of groundwater quality for drinking usage: A case study of Southern Haryana, India. Environmental Geology, 54(2), 249-255. doi:10.1007/s00254-007-0812-9

Srivastava. (2019). Fuzzy Models for Water Quality Assessment. 10.1109/ICICT46931.2019.8977693

Walaa, A., \& Mona, G. (2019). Assessing the Agricultural Drainage Water with Water Quality Indices in the El-Salam Canal Mega Project, Egypt. Water (Basel), 11(5), 1013. doi:10.3390/w11051013

Wang, M., Wang, X., Liu, Q., Shen, F., \& Jin, J. (2020). A novel multi-dimensional cloud model coupled with connection numbers theory for evaluation of slope stability. Applied Mathematical Modelling, 77(Jan), 426-438. doi:10.1016/j.apm.2019.07.043

Wei-X., L., Zhang, X.-X., Wu, B., Sun, S.-L., Chen, Y.-S., Wen-Yang, P. A. N., Zhao, D.-Y., \& Cheng, S.-P. (2008). A Comparative Analysis of Environmental Quality Assessment Methods for Heavy Metal-Contaminated Soils. Pedosphere, 18(3), 344-352. doi:10.1016/S1002-0160(08)60024-7

Wen-Bo, F., Xiao-Juan, L. I., Hai-Juan, W., Yu, J., Gang, L. I., \& Chang-Lu, Q. (2018). Recognition and practice of professional certification in agricultural water conservancy engineering. Education Teaching Forum.

Xue, L. I., Liu, Y., \& Guo-Xin, Q. I. (2018). Research on temporary project fees in agricultural water conservancy quota based on gray fuzzy theory. Journal of Anhui Agricultural.

Yadav \& Deshpande. (2021). Risk-Based Optimal Ranking of Air Quality Monitoring Stations in a Fuzzy Environment: A Case Study. 10.1007/978-981-15-5511-4_14

Zamani, R., Ali, A. M. A., \& Roozbahani, A. (2020). Evaluation of adaptation scenarios for climate change impacts on agricultural water allocation using fuzzy mcdm methods. Water Resources Management: An International Journal, 34.

Zhang, J., Song, W., Jiang, B., \& Li, M. (2018). Measurement of lumber moisture content based on PCA and GS-SVM. J. For. Res., 29(2), 557-564. doi:10.1007/s11676-017-0448-x

Zhang, W., Kantor, G., \& Singh, S. (2004). Integrated wireless sensor/actuator networks in an agricultural application. Proceedings of the 2nd international conference on Embedded networked sensor systems - SenSys '04, 17.

Zheng, X., Li, Y., Liu, Z., \& Wang, C. (2019). Steady-state control strategy of VSC-HVDC transmission system based on full-order terminal sliding mode control method. Journal of Engineering (Stevenage, England), 987-990(16), 987-990. Advance online publication. doi:10.1049/joe.2018.8463

Zobel, R. W., \& Lychalk, R. F. (1999). Aeroponic growth system with nutrient fog stabilization. US Patent US5937575A.

Shen Shan Han is associated with School of Resource Environment and Historical Culture, Xianyang Normal University, Xianyang 712000, China. They have expertise in mathematical modeling, fuzzy logic etc. Han has been working for various projects and given services as a reviewer to various reputed journals. 